\title{
MRI and Stereo Vision Surface Reconstruction and Fusion
}

\author{
Trishia El Chemaly \\ Department of Biomedical Engineering \\ Holy Spirit University of Kaslik \\ Kaslik, Lebanon \\ trishia.c.elchemaly@net.usek.edu.lb
}

\author{
Vincent Groenhuis \\ Department of Electrical Engineering, \\ Mathematics and Computer Science \\ University of Twente \\ Enschede, The Netherlands \\ v.groenhuis@utwente.nl
}

\author{
Françoise J. Siepel \\ Department of Electrical Engineering, \\ Mathematics and Computer Science \\ University of Twente \\ Enschede, The Netherlands \\ f.j.siepel@utwente.nl
}

Ferdi van der Heijden

Department of Electrical Engineering, Mathematics and Computer Science University of Twente

Enschede, The Netherlands

f.vanderheijden@utwente.nl

\author{
Sandy Rihana \\ Department of Biomedical Engineering \\ Holy Spirit University of Kaslik \\ Kaslik, Lebanon \\ sandyrihana@usek.edu.lb
}

\author{
Stefano Stramigioli \\ Department of Electrical Engineering, \\ Mathematics and Computer Science \\ University of Twente \\ Enschede, The Netherlands \\ s.stramigioli@utwente.n
}

\begin{abstract}
Breast cancer, the most commonly diagnosed cancer in women worldwide, is mostly detected through a biopsy where tissue is extracted and chemically examined or pathologist assessed. Medical imaging plays a valuable role in targeting malignant tissue accurately and guiding the radiologist during needle insertion in a biopsy. This paper proposes a computer software that can process and combine 3D reconstructed surfaces from different imaging modalities, particularly Magnetic Resonance Imaging (MRI) and camera, showing a visualization of important features and investigates its feasibility. The development of this software aims to combine the detectability of MRI with the physical space of the camera. It demonstrates that the registration accuracy of the proposed system is acceptable and has potential for clinical application.
\end{abstract}

Keywords—breast cancer; biopsy; MRI; stereo vision

\section{INTRODUCTION}

Breast cancer is the most commonly diagnosed cancer in women worldwide and the second major cause of cancer death among women [1].

A biopsy is the only diagnostic procedure that can confirm whether the suspicious area is cancerous or not. During this test, tissue is removed from the suspicious area for examination [2] guided by either real-time ultrasound or MRI. During MRI breast biopsy, the patient is taken out of the MRI machine after imaging and then taken back in after the needle has been placed to check for correct placement. Sometimes, multiple repetitions of this procedure are needed before the needle has been found to have been inserted correctly, increasing patient discomfort, time, cost, and the number of false negative diagnoses.

Medical image registration could be a valuable assistant for experts in such interventions where a target image is aligned to a reference image in order to create a more comprehensive output image and achieve accurate instrument placement, eliminating the need for multiple needle insertion. A key application in this case is physical- to-image space registration where camera images are aligned to medical images in order to guide physicians or biopsy robots. [1].

The main focus of this paper is the development of a computer software that can reconstruct the surface of breast phantoms imaged with different modalities, particularly MRI and stereo camera then fuse the multiple surfaces. This fusion should allow the visualization and localization of important features, specifically markers. The ultimate goal is to combine MRI lesion detectability with stereo vision localization in the physical space in order to achieve accurate needle placement and tissue extraction in the future. The software deals with the segmentation, registration, and transformation of MRI scans and camera images of the breast. It should promote exact targeting of small lesions and particular features to improve clinical workflow.

\section{PREVIOUS WORKS}

Over time, diverse methods of image fusion were presented. In extrinsic methods, clearly visible markers are attached to the patient with the necessity of being accurately detectable in all the acquired modalities. Traditionally, markers are placed on the skin and extracted manually or automatically with detection methods. Reference [3] used stereo vision to calculate coordinates of markers in physical space. The upper hand of depth cameras remains in their ability to image in real time and acquire intensity or color images simultaneously [4].

\section{IMPLEMENTATION}

This work was applied on breast phantoms with 12 circular 3D printed markers filled with petroleum jelly that is MRI visible. It comprises four modules.

\section{A. MRI Surface Reconstruction}

The aim of this module was to create a 3D surface from MRI images of the phantom and localize the markers, to later assist in the registration. 
1) Image Enhancement: The DICOM (Digital Imaging and Communications in Medicine) images were enhanced with bright stretching.

2) Image Binarization: The enhanced images were binarized using Otsu's method which comprises an exhaustive search for the threshold that minimizes the intra-class variance.

3) 3D Volume Visualization: The isosurface method was used to visualize the 3D MRI breast by connecting points that are above a certain threshold chosen as 0.5 due to interest in white pixels.

4) Markers Localization: To begin, all objects in the $3 \mathrm{D}$ volume detected based on 26-connectivity. The 12 markers were segmented out of the blocks based on the area. The centroids of the markers were transformed from image coordinates $(\mathrm{px})$ to anatomical coordinates $(\mathrm{mm})$ by an affine transformation.

\section{B. Design of the Camera Setup}

A camera set-up was envisioned in order to localize important features. The setup consisted of two Matrix Vision BlueFox- IGC USB 2.0 cameras mounted on the end effector of a robot arm. The requirements included a surface reconstruction accuracy of $1 \mathrm{~mm}$, a $25 \mathrm{~cm}$ field of view, a working range of 40 to $70 \mathrm{~cm}$, and a maximum baseline of 15 $\mathrm{cm}$ in order to fit the robot arm's end effector.

1) Choice of Lenses: Taking into consideration a camera sensor size s of $4.8 \mathrm{~mm}$, a field of view FOV of $250 \mathrm{~mm}$, and a minimum working distance Dmin of $400 \mathrm{~mm}$, the focal length $\mathrm{f}$ of the lens to be used is calculated as $8 \mathrm{~mm}$ with

$$
f=s^{*} \operatorname{Dmin} / F O V
$$

2) Distance Range Analysis: In optics, the depth of field (DOF) is the range of distance from the camera in which an object can be photographed and still yield a focused image. Even if a lens can only be focused at one distance at a time, the decline in sharpness is gradual, in a way that within the DOF, the unsharpness is imperceptible.

The diameter of the circle of confusion or blur circle can be calculated based on the following equation.

$$
c=A * f *(D 1-D 2) /(D 1-f) * D 2
$$

A : Aperture diameter

D1: In focus working distance

D2: Current working distance

f : Focal length

3) Choice of Camera Setup: A parallel stereo camera setup allows the maximization of the overall FOV covered by both cameras but has a limited overlap between the FOVs of the two cameras. On the other hand, a convergent camera setup would be optimal in an indoor application where utility of the camera visual range is maximized. In addition, according to [5], as the verging angle between the stereo cameras increases, accuracy is improved. For an optimal setup in this project, the cameras must be verging.

4) Camera Locations: The locations of the cameras can be defined by the baseline and verging angle $\theta$.

$$
\theta=\arctan (b / D \min )
$$

5) Monte Carlo Analysis: A Monte Carlo simulation has been performed to test the reliability of a camera setup based on its characteristic baseline $b$.

The model whose reliability is to be tested is a converging camera setup with $b$ and $\theta .100$ 3-dimensional points are considered to be imaged by two cameras. The image coordinates of these points in the two camera coordinate systems are known. An algorithm is written in order to estimate the corresponding 3D world coordinates of these 100 points based on a homography applied to their 2D image coordinates.

The 3D coordinates are estimated for a number of algorithm repetitions $\mathrm{N}_{\mathrm{MC}}=200$ where for each repetition, a random noise is added to both images. Finally, the covariance of the resulting estimates obtained over the 200 repetitions is used as a measure of the reliability of the model. The simulation is applied on several models with different characteristic baselines.

\section{Camera Surface Reconstruction}

The aim of this part is to create a 3D surface from camera acquired images of the breast phantom and localize markers placed on the phantom to later assist in the registration. Stereo vision techniques were used in the process [6].

In stereo vision, two cameras acquire images of the same scene, but they are separated by a distance - exactly like our eyes. The shift between the images is called the disparity and is used to calculate objects' distance from the camera setup.

To be able to match the images and eventually calculate the disparity and depth, the position of one camera must be accurately defined with respect to the second. For that, camera calibration is needed as the first step of the whole process.

1) Stereo Camera Calibration: First, it is essential to calibrate the two cameras, which means define the location and orientation of the second camera with respect to the first.

2) Stereo Rectification: It allows to virtually align the 2 cameras to facilitate epipolar geometry. Simply, the image from the second camera is rectified by undoing its rotation with respect to that of the first and aligned to the baseline of the first camera.

3) Stereo Matching and Disparity Map: Stereo matching constitutes finding the corresponding points in 2 images. Next, the disparity or spatial shift could be computed between all corresponding pixels.

Sometimes, point clouds are too scarse in data points due to reconstruction from disparity maps with few reliable points. In that case, the $3 \mathrm{D}$ data points are insufficient to create a dimensional reconstruction. Since the disparity map is obtained from the matched features in the two images, an adequate stereo matching method must be chosen. A Speeded Up Robust Features (SURF) detector has been used in order to boost the stereo matching.

4) Triangulation: Triangulation is the principle of estimating depth from images taken from two different points of view. Knowledge of the length of the base line and the two angles formed by the base line and the two rays from the 3D point, suffices to calculate the depth of the 3D point and then its $\mathrm{X}, \mathrm{Y}$, and $\mathrm{Z}$ coordinates which are function of the disparity. 
5) Marker Localization: Similarly to the final step in MRI reconstruction, the breast phantom markers must be localized for use in registration. What characterizes the markers used is their color that can be defined as hue, saturation, and value (HSV). A mask was created based on the HSV characteristics of the markers and applied to the breast image in the aim of segmenting the markers. Once the markers are localized in 2D in a pair of corresponding images, they are triangulated to obtain their 3D coordinates in $\mathrm{mm}$.

\section{MRI-Camera Registration}

3D registration was applied on MRI and camera reconstructed surfaces. The camera reconstructed surface was chosen as the reference volume since it depicts the relative position of the breast to the camera, and hence the robot. A geometrical transformation should be obtained to map the target surface (MRI surface) to the reference surface.

A common approach for 3D surface registration is the iterative closest point (ICP) algorithm which finds the transformation between 2 point clouds by minimizing the square errors. It then assumes closest points correspond to each other and computes the best transform. The ICP is only applicable in the case where a good first estimate of transformation is available. It could be then used to optimize the process.

For the first transformation, the centroids of the markers placed on the breast phantom could be used. The first transformation between the two surfaces can be estimated by transforming the MRI markers to the Camera markers using Procrustes, a geometric transformation that only involves translation, rotation, uniform scaling, or a combination of these. The rotation and translation are defined such that the two objects are superimposed and their shapes will be compared. The objective is to obtain a similar placement and size, by minimizing a measure of shape difference called the Procrustes distance between the objects.

\section{RESULTS}

\section{A. MRI Surface Reconstruction}

The 3D MRI surface was obtained in anatomical space as seen in Figure 1, and the markers' centroids were localized.

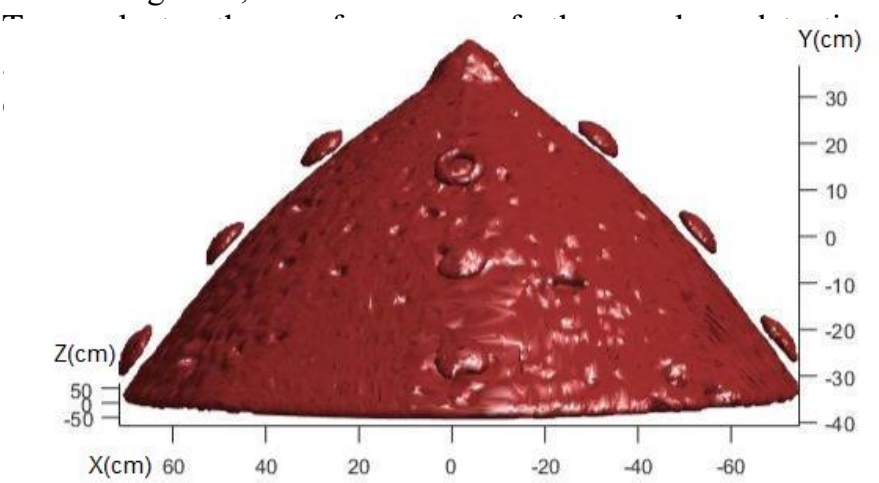

FigURE 1. 3D VOLUME VISUALIZATION OF THE BREAST PHANTOM

\section{B. Design of the Camera Setup}

Two Matrix Vision BlueFox-IGC USB 2.0 cameras were mounted with $8 \mathrm{~mm}$ lenses and placed converged to each other. The results of the Monte Carlo analysis showed that a model with a larger baseline is more likely to be more reliable for obtaining 3D coordinates and thus for 3D reconstruction as shown in Table 1. As a first design, the camera holder was printed with $5.5 \mathrm{~cm}$ baseline and $7.9^{\circ}$ verging angle (3).

TABLE 1. COVARIANCE IN 3D COORDINATES ESTIMATION AS A FUNCTION OF BASELINE

\begin{tabular}{|c|c|}
\hline Baseline b $(\mathbf{c m})$ & Covariance in z coordinate \\
\hline 5 & 11.86 \\
\hline 10 & 2.84 \\
\hline 15 & 1.19 \\
\hline 20 & 0.65 \\
\hline 25 & 0.38 \\
\hline
\end{tabular}

\section{Camera Surface Reconstruction}

The calibration session gave a reprojection error of 0.14 pixels. Knowing that the pixel dimension for the camera used is $6 \mu \mathrm{m}$, this implies that the reprojection error is about $0.14 \times 6$ $=0.84 \mu \mathrm{m}$.

Experimentation proved that using SURF and range definition improved the disparity map as seen in Figure 2.
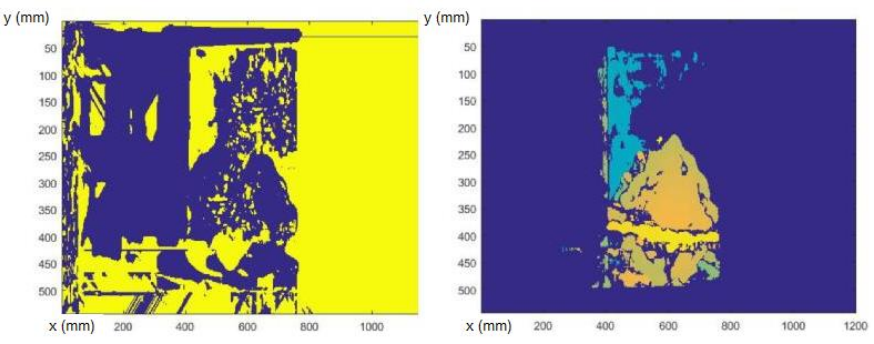

Figure 2. DisPARITY MAP IMPROVED

The breast phantom was then reconstructed from the disparity map as seen in Figure 3.

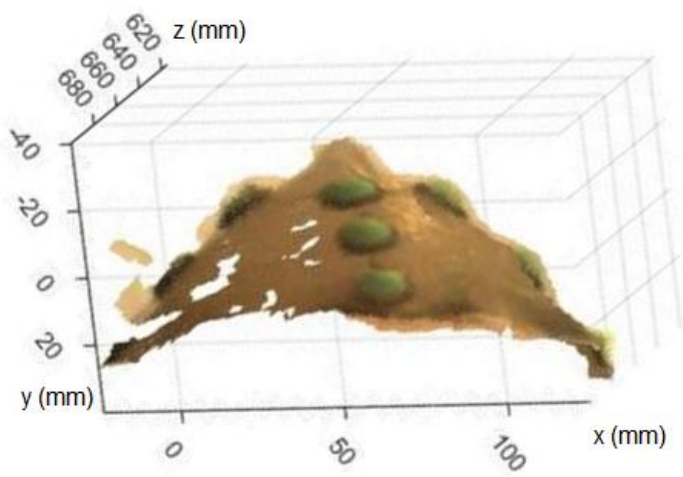

FIGURE 3. RECONSTRUCTION OF THE BREAST PHANTOM 


\section{MRI-Camera Registration}

Figure 4 shows the results of the registration approach on 2 camera point clouds before and after applying ICP.
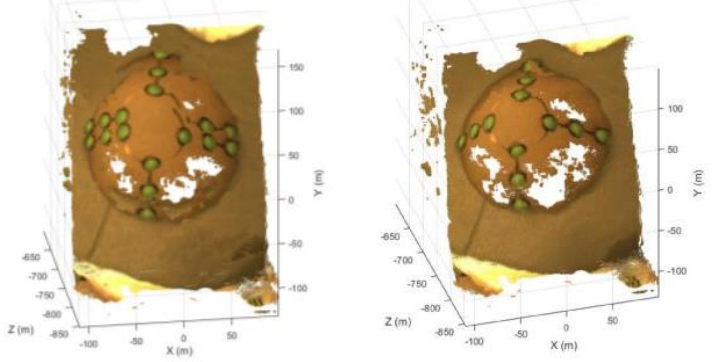

Figure 4. REgisTRATION RESULTS BEFORE AND AFTER APPLYING ICP

Figure 5 shows the final camera-camera surface registration result from 6-point clouds.

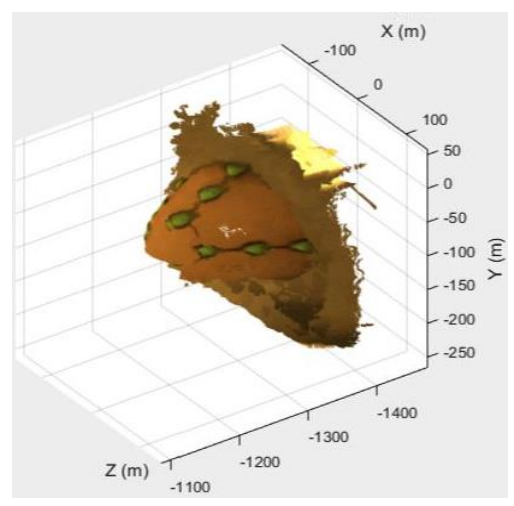

Figure 5. Final Registration RESUlt (CAMERA-CAMERA) FROM 6 POINT CLOUDS

The results of the final MRI-camera registration are illustrated in Figure 6.

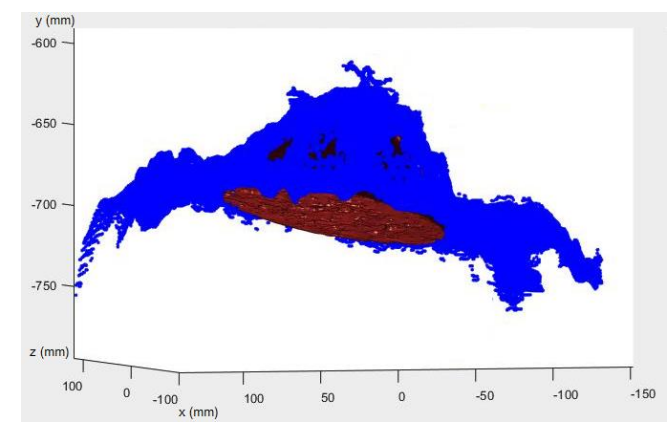

FIGURE 6. MRI-CAMERA REGISTRATION

The RMSE calculated from the transformation was reduced from $7.75 \mathrm{~mm}$ to $3.39 \mathrm{~mm}$ when the outliers were excluded from the registration.

\section{CONCLUSION}

This study was conducted in the aim of testing the applicability and accuracy of MRI-Camera surface registration in the context of robotic assisted breast biopsies. A computer software that can reconstruct and fuse the surface of breast phantoms imaged with the two modalities was developed.
In MRI surface reconstruction, an average error of $0.5 \mathrm{~mm}$ in marker localization was considered as acceptable.

The camera setup implemented with rapid 3D printed prototyping helped achieve the project goals.

In camera surface reconstruction, experiments proved that SURF is an important tool for optimizing disparity maps and improving the reconstruction to finally obtain satisfactory results. The color-based method proposed for localizing markers in real space was successful in different lighting conditions.

As for registration, experimentation results showed that the accuracy of the algorithm (average of $3.39 \mathrm{~mm}$ for cameracamera registration) is reasonably acceptable for a first prototype and competes with an existing Kinect-based CTcamera registration approach with mean target positioning error of $5.23 \mathrm{~mm} \mathrm{[7].}$

For the future, the use of markers that could be more accurately localized could improve the registration accuracy. It is also predicted to elaborate the algorithm in the aim of detecting lesions and evaluating the error in that case.

The registration of MRI and Camera reconstructed surfaces offers countless promises in what concerns combining the accuracy of MRI detectability and camera guidance for exact needle steering and a robust biopsy. In this context, a novel study was conducted and presented an impressive potential for clinical application of MRI-camera registration and the integration of medical imaging in robotic systems for cancer diagnostic operations.

\section{ACKNOWLEDGEMENTS}

This project has received funding from the European Union's Horizon 2020 research and innovation programme under grant agreement No 688188.

\section{REFERENCES}

[1] "Breast Cancer Facts," 2016. [Online]. Available: http://www.nationalbreastcancer.org/.

[2] "Breast Cancer Biopsy," 2016. [Online].

[3] S. Nicolau, A. Garcia, X. Pennec, L. Soler and N. Ayache, "An augmented reality system to guide radio-frequency tumour ablation," Computer Animation and Virtual Worlds, vol. 16, no. 1, pp. 1-10, 2005.

[4] L. Maier-Hein, A. Tekbas, A. Seitel, A. Müller, F. Pianka, S. Satzl, S. Schawo, B. Radeleff, R. Tetzlaff and A. Tetzlaff, "In vivo accuracy assessment of a needle-based navigation system for CT-guided radiofrequency ablation of the liver," Medical Physics, vol. 35, p. 538596, 2008.

[5] Deqiang Xiao, Huoling Luo, Fucang Jia, Yanfang Zhang, Yong Li, Xuejun Guo, Wei Cai, Chihua Fang, Yingfang Fan, Huimin Zheng and Qingmao $\mathrm{Hu}$, "A Kinect ${ }^{\mathrm{TM}}$ camera based navigation system for percutaneous abdominal puncture," Physics in Medicine and Biology, vol. 61 , no. 15,2016 [6] F. V. d. Heijden, "3D surface reconstruction," University of twente -
RAM, p. 3, 2016.

[7] T. Pribanic, M. Cifrek and S. Tonkovic, "The Choice of Camera Setup in $3 \mathrm{D}$ Motion Recinstruction Systems," in The 22"' Annual EMBS International Conference, Chicago IL, 2000. 